\title{
Seeking patient's consent for examination and treatment: Introducing a consent form to be used in government hospitals in Sri Lanka.
}

\author{
Dhananjaya L. Waidyaratne \\ Department of Forensic Medicine, Teaching Hospital Anutradhapura
}

\section{Background}

Respect of the patient's right to self determination (autonomy), through informed consent is considered the foundation of doctorpatient relationship.

Despite the considerable development in health infrastructure and human resources, a regular practice of modern ethical standards has not yet been appropriately established. So far the country has failed even to reach a consensus regarding informed consent. Frequently the "consent for medical examination and treatment" was looked from a paternalistic view point, often only as a tool of defense against litigation. Obtaining consent was merely limited to getting any person, accompanying the patient to hospital, to sign on bed head ticket (BHT), next to a "stereotype" phrase: "No property, consent for operation". Usually no doctors were involved in this process, a nurse, receiving patient to the ward, write the phrase. Patient information about the nature and consequences of procedures or available treatment options were often ignored.

The situation is being changed to the better. Many doctors are exploring ways of obtaining consent; formats are being designed by individual clinicians. Incompleteness in view of legal validity and unsuitability for use in different situations were among many shortfalls noted in those consent forms.

The purpose of this paper is to focus on practical medico legal issues concerning consent and clinical ethics and to propose a format which may be appropriate and applicable in many of the clinical scenarios for obtaining consent.

\section{Main Objective}

The main objective was to design a suitable format to obtain informed consent of patients for various surgical, investigative and therapeutic procedures in clinical practice.

\section{Specific objectives}

1. Identify the quantity and the quality of information, that patients should be given in order for the consent to be valid

2. Design a widely acceptable format to obtain informed consent of patients for various surgical, investigative and therapeutic procedures in clinical practice

\section{Ethical principles concerning the informed consent}

Patient consent is required on occasions where the doctor wishes to initiate an examination or treatment or any other intervention, except in emergencies or where the law prescribes otherwise (such as where compulsory treatment is authorized by mental health legislation). Consent may be explicit or implied. Explicit (or expressed) consent is when a person actively agrees, either orally or in writing. Implied consent is when signaled by the behavior of an informed patient. Implied consent is not considered a lesser form of consent but it only has validity if the patient genuinely knows and understands what is being proposed

Failure to follow the recommended protocols for obtaining meaningful, lawful consent is unethical and can harm patients both physically and psychologically. 
Interventions without obtaining consent can end in either civil litigation or in rare cases, criminal prosecution. A quality health care demands more than mere technical proficiency, and wherever possible it needs to allow for active patient participation. This cannot be achieved in an atmosphere of mistrust, or if patients feel that what they think and say is of no real consequence.

Consent is a process, not a one-off event, and it is important that there is continuing discussion to reflect the evolving nature of treatment. A consent form simply documents that some discussion about the procedure or investigation has taken place. It is only an evidence of a process, not the process itself.

The provision of sufficient accurate information is an essential part of seeking consent. Competent adult patients are entitled to refuse consent to treatment, even when doing so may result in permanent physical injury or death. Compliance when a patient does not know what the intervention entails, or is unaware that he or she can refuse, is not 'consent'. Doctors must respect a refusal of treatment if the patient is an adult who is competent, properly informed and is not being coerced.

The doctor who recommends that the patient should undergo the intervention should have responsibility for providing an explanation to the patient and obtaining his or her consent. In a hospital setting this will normally be the senior clinician. In exceptional circumstances the task of reaffirming consent can be delegated to a doctor who is suitably trained and qualified, is sufficiently familiar with the procedure and possesses the appropriate communication skills.

Any discussion, however, should be recorded in the patient's medical notes.

Generally there is no legal requirement to obtain written consent but in some cases it may be advisable.
Extent of patient information required for a valid consent

The information that patients should be provided with, includes,

- Purpose of the investigation or treatment, details and uncertainties of the diagnosis,

- Options for treatment, including the option not to treat, explanation of the likely benefits and probabilities of success for each option the risks such as known possible side effects,

- Complications and adverse outcomes including where intervention and/or treatment may fail to improve the condition,

- The name of the doctor who will have overall responsibility,

- A reminder that the patient can change his or her mind at any time.

A careful balance needs to be struck between what patients want to know and ought to know (i.e. listening to what the patient wants and providing enough information) in order that the patient's decisions are informed

\section{Introducing the proposed consent form}

The proposed consent form is believed to be appropriate for obtaining written consent from patients for examination and treatment in government hospitals in Sri Lanka. The development of this format took more than 03 years of consultation and discussion with different medical/surgical specialists attached to Matale and Anuradhapura hospitals during the period between 2008 and 2010.

Efforts had been made to make the form to be complete and comprehensive while being concise to be user-friendly.

- Important information referring to identity of the patient and nature of the proposed intervention is included in the given format. 
- Further it elaborates the areas of most valuable concern in the discussion leading to informed consent, though it does not contain the concrete constituent facts.

- The document guides both clinicians and patients into their rights and responsibilities.

(Singhalese/English and Tamil/English versions of proposed consent form are given below.)

Since the amount of information which, doctors should provide to each patient will vary according to factors such as the nature and severity of the condition, the complexity of and the risks associated with the treatment or procedure and the patient's own wishes, it is simply impossible to design a universal consent form carrying all necessary information. As such this consent form needs to be supplemented with provision of information applicable to respective areas of medical practice or disciplines, clinical scenarios, individual interventions and procedures. Good quality information leaflets that patients can take away with them can be a useful way of improving information provision but these should not be seen as an alternative to discussion.

Separate Sinhalese/English and Tamil/English versions were thought to be justifiably appropriate to use for any patient. 


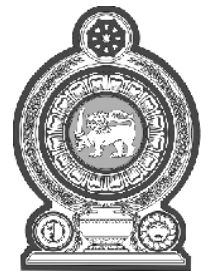

๔రౌ๋ఠอ

மாவட்ட பொது

Hospital

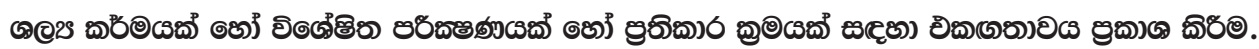

eclaration of Consent for Surgical or Special Investigative or Therapeutic Procedure.

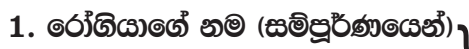

Name of Patient (in full) $\}$

2. อณ®

Age $\}$

\begin{tabular}{|l|c|}
\hline gorø & జేశ్ర \\
Male & Female \\
\hline
\end{tabular}

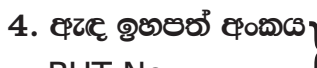

$\left.\begin{array}{l}\text { 5. อృరิอ઼อ } \\ \text { Ward }\end{array}\right\}$

BHT No.

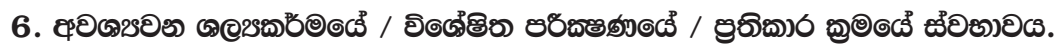

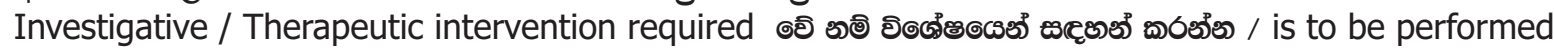
\& Nature of the Surgical

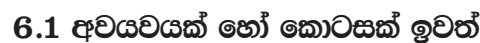

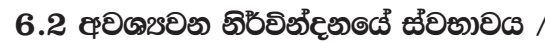

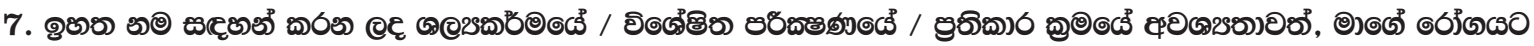

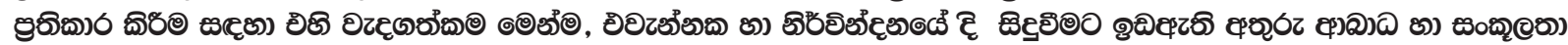

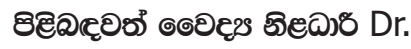

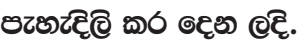

Explained me about the necessity, and importance of the above mentioned procedure in treating my illness and possible risks and complications of such a procedure and anaesthesia.

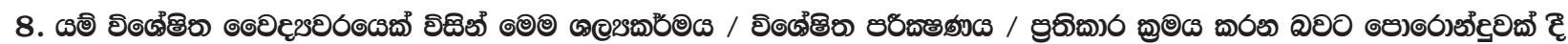

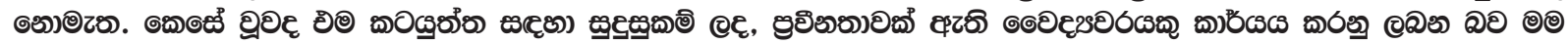
દृకిత్. I have never been promised that a particular doctor would do this procedure. However, I understand that a doctor with necessary qualification, skill and proficiency would be attending to the procedure.

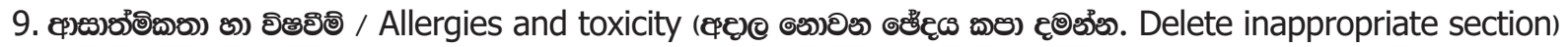

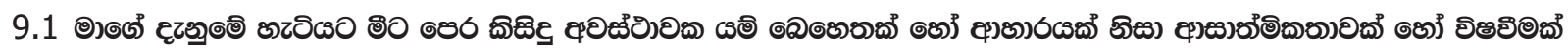

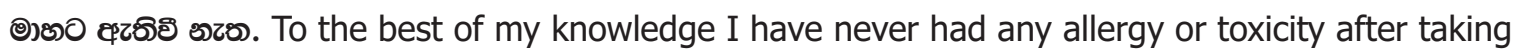
any drug or food

9.2

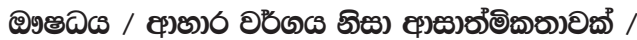

రిశతిఅదో అృరర ణఁరి రిద. I have experienced an allergic / a toxic reaction to following drug / food item

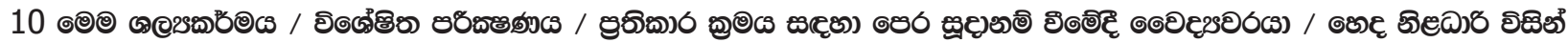

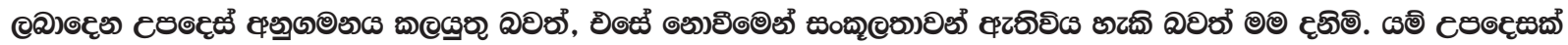

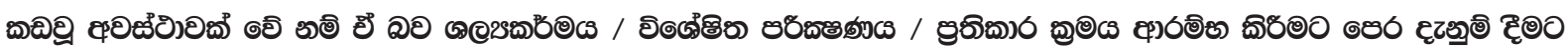
లిఐఁ ఠอతి. I understand that I must strictly follow the instructions given by the Doctor / Nurse while preparing for the procedure and I understand that any breach would result in complications. I undertake the responsibility of informing of such breach if any before commencement of the procedure. 
Seeking patient's consent for examination and treatment: Introducing a consent form to be used in government hospitals in Sri Lanka.

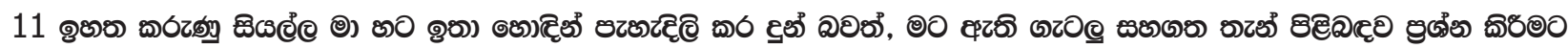

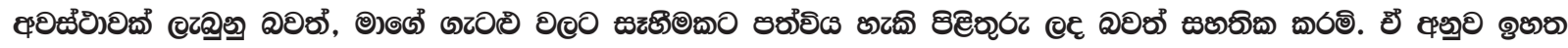

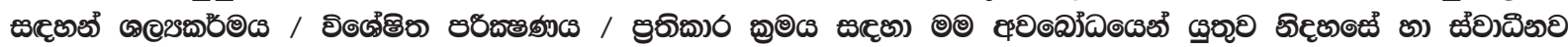

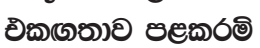

I certify that everything mentioned above was explained to me carefully and I was given the opportunity to question and clarify all my queries to my satisfaction. Accordingly, I express my free and voluntary informed consent for the above mentioned procedure.

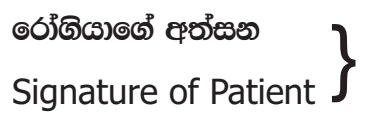

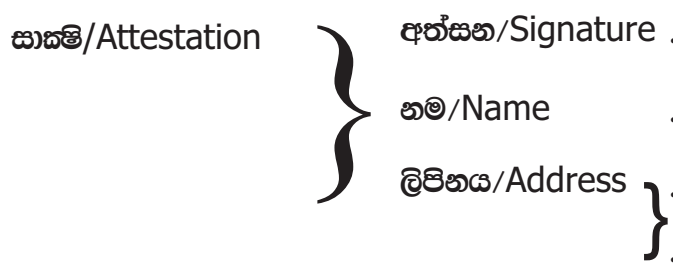

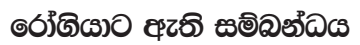

Relationship to the Patient $\}$

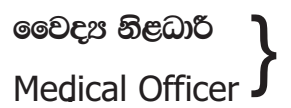

$\left.\begin{array}{l}\text { ¿ृฒn } \\ \text { Date }\end{array}\right\}$ 


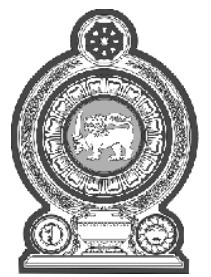

\section{๑రౌ்ల}

மாவட்ட்ட பொது

Hospital

சத்திரசிகிச்சை அல்லது விசெட பரிசொதனை அல்லது சிகிச்சைக்காக இணக்கம் டிதரிவித்தல்

Declaration of Consent for Surgical or Special Investigative or Therapeutic Procedure.

1. நொயாளியின் டிபயர் (முபுமையாக) \} Name of Patient (in full)

\}

$\left.\begin{array}{l}\text { 2. வயது } \\ \text { Age }\end{array}\right\}$

\begin{tabular}{|c|c|}
\hline டிபண் & ஆண் \\
Female & Male \\
\hline
\end{tabular}

$\left.\begin{array}{l}\text { 4. கட்ழல் இலக்கம் } \\ \text { BHT No. }\end{array}\right\}$

$\left.\begin{array}{l}\text { 5. வாட்டு } \\ \text { Ward }\end{array}\right\}$

6. அவசியப்படும் தத்திரசிகிச்சையின் / விசெட பரிசொதனையின் / சிகிச்சையின் தன்மை Nature of the Surgical / Investigative / Therapeutic intervention required. (ஏதாவது அவையவ“களை அல்லது பகுதியை அகற்றல் அல்லது டிபாருத்துதல் பற்றி விசெடமாகக் குறிப்பிடவஜம்) / please specifically state if removal of any organ or part or any grafts is to be performed)

6.1 அவயவ“கள் அல்லது பகுதியை அகற்றல் அல்லது இணைத்தல் /Removals or grafts

6.2 தெவையான மயக்கவியலின் தன்மை / Nature of anaesthesia required.

7. மெலெ டிபயர் குறிப்பிடப்பட்ட சத்திரசிகிச்சையின் / விசெட பரிசொதனையின் /சிகிச்சை முறையின் அவசியமும் முக்கியத்துவமும் மற்றும் மயக்கவியலினால் ஏற்படக்வுழய இடர்பாடுகள் பற்றியஜம் தீமைகள் பற்றியஜம் வைத்திய

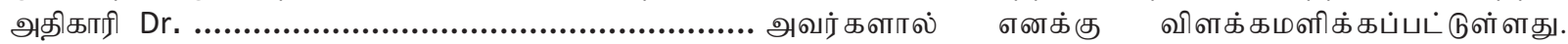
explained me about the necessity, and importance of the above mentioned procedure in treating my illness and possible risks and complications of such a procedure and anaesthesia.

8. யாராவது டிபயர் குறிப்பிட்ட விசெட வைத்தியரால் இந்த சத்திரசிகிச்சை / விசெட பரிசொதனை/ சிகிச்சை முறை டிசய்யப்படுவதாக உத்தரவாதம் அளிக்கப்படவில்லை. எவ்வாறாயினும் அந்த விடயத்தினை தகைமை டிபற்ற நிபஜணத்துவம் ஜடிபற்ற வைத்தியரால் அவ்விடயம் நிறைவெற்றப்படும் என்பை நான் அறிவென; I have never been promised that a particular doctor would do this procedure. However, I understand that a doctor with necessary qualification, skill and proficiency would be attending to the procedure.

9. ஒவ்வாமை அல்லது விசமாதல் கூ யுடடநசபநைள யனெ வழஒவைவைல (தெவையற்ற விடயத்தை டிவட்ழவிடவஜம்.). Delete inappropriate section)

9.1 எனது அறிவஜக்கு எட்ழய விதத்தில் இதற்கு முன் எந்தடிவாரு சந்தர்ப்பத்திலும் ஏதாவது மருந்து அல்லது உணவஜ வகையால் ஒவ்வாமை அல்லது விசத்தன்மை ஏற்படவில்லை. To the best of my knowledge I have never had any allergy or toxicity after taking any drug or food.

9.2 சாப்பிட்டால் ஒவ்வாமைத் தன்மை $\therefore$ விசமாகுதல் எனக்கு ஏற்படுவது உண்டு. I have experienced an allergic / a toxic reaction to following drug / food item 
10. இந்த சத்திரசிகிச்சை / விசெட பரிசொதனை / சிகிச்சை முறைக்கு முன்னாயத்தமாகும் பொது வைத்த ியர/ தாதியர்களால் வர்“கப்பட்ட ஆலொசனைகள் கட்டாயமாக பின்பற்றப்பட வெண்ழயதன் அவசியத தையஜம் அவ்வாறு இல்லாத சந்தர்ப்பத்தில் பாதிப்பஜக்கள் ஏற்படும என்றும் நான் அறிவென்.ஏதாவது ஆலொசனைகள் மீீற்ப்படுமானால் அதனை சத்திரசிகிச்சை / விசெட பரிசொதனை முறை ஆரம்பிப்பதற்கு முன் அவற்றைச் டிசால்வதற்கு நான் உடன்படுகின்றென்.

I understand that I must strictly follow the instructions given by the Doctor / Nurse while preparing for the procedure and I understand that any breach would result in complications. I undertake the responsibility of informing of such breach if any before commencement of the procedure.

11 மெற்குறிப்பிடப்பட்ட எல்லா விடய“களையஜம் மிக நன்றாக எனக்கு விளக்கியஜள்ளதொடு எனக்கு உள்ள ஐய“கள் சம்பந்தமாக நான் கெள்வி கெட்பதற்கு சந்தர்ப்பம் வர்“கப்படுள்ளதொடு எனது கெள்விகளுக்கு திருப்தி அடையக்வுழயவாறு விடைகள் டிபறப்பட்டுள்ளதாகவஜம் உறுதி வுறுகின்றென். அதனழப்படையில் மெற்குறிப்பிட்ட சத்திரசிகிச்சை / விசெட பரிசொதனை / பரிசொதனை முறைக்காக எனது அறிவஜக்கு எட்ழய வகையில் சுதந்திரமாகவஜம் மற்றும் சுயாதீனமாகவஜம் உடன்படுகின்றென். I certify that everything mentioned above was explained to me carefully and I was given the opportunity to question and clarify all my queries to my satisfaction. Accordingly, I express my free and voluntary informed consent for the above mentioned procedure.

$\left.\begin{array}{l}\text { நொயாளியின் பெயர் } \\ \text { Signature of Patient }\end{array}\right\}$

சாட்சி/Attestation $\left\{\begin{array}{l}\text { கைடியாப்பம் /Signature . } \\ \text { டிபயர் / Name } \\ \text { விலாசம் / Address }\end{array}\right\}$

$\left.\begin{array}{l}\text { நொயாளியஜடனான உறவஜமுறை } \\ \text { Relationship to the Patient }\end{array}\right\}$

$\left.\begin{array}{l}\text { வைத்திய அதிகாரி } \\ \text { Medical Officer }\end{array}\right\}$

திகதி/Date \} 


\section{Acknowledgment}

Many of the Consultants attached to District General Hospital, Matale and Teaching Hospital, Anuradhapura, during the period between 2008 and 2010 contributed with their ideas to the development of this consent form. Tamil translation of the consent form was done by Mr. N.P.A. Rahuman, Teacher attached to Zahira College, Anuradhapura and Dr. U. Mayorathan. Services of all of them should be highly commended.

\section{Reference}

1. Mason J.K., McCall Smith R.A. , Laurie G.T. Law and Medical Ethics. $5^{\text {th }}$ edn. London: Butterworths. (1999). pp 244-288

2. Plueckhahn Vernon D. Cordner Stephen M. Ethics Legal Medicine and Forensic Pathology. $2^{\text {nd }}$ edn. Melbourne University Press (1991).pp 1-5

3. Wilks M., Kinght M. The Practitioner's Obligations in Clinical Forensic Medicine. McLay WDS $2^{\text {nd }}$ edn. London, Greenwich Medical Media, 1996, Chap. 3 pp 43-45.

4. Worthington R. (2002) Clinical issues on consent: Some philosophical concerns J. Medical Ethics; 28: 377-380

5. Consent tool kit, British Medical Association downloaded from the website www.bma.org.uk/ethics 